Portland State University

PDXScholar

Engineering and Technology Management

Faculty Publications and Presentations

$7-2006$

\title{
Fuzzy Cognitive Maps for Engineering and Technology Management: What Works in Practice?
}

Antonie J. Jetter

Portland State University, ajetter@pdx.edu

Follow this and additional works at: https://pdxscholar.library.pdx.edu/etm_fac

Part of the Engineering Commons

Let us know how access to this document benefits you.

\section{Citation Details}

Jetter, A.J., "Fuzzy Cognitive Maps for Engineering and Technology Management: What Works in Practice?," Technology Management for the Global Future, 2006. PICMET 2006, vol.2, no., pp.498,512, 8-13 July 2006 doi: 10.1109/PICMET.2006.296648

This Conference Proceeding is brought to you for free and open access. It has been accepted for inclusion in Engineering and Technology Management Faculty Publications and Presentations by an authorized administrator of PDXScholar. Please contact us if we can make this document more accessible: pdxscholar@pdx.edu. 


\title{
Fuzzy Cognitive Maps for Engineering and Technology Management: What Works in Practice?
}

\author{
Antonie J. Jetter \\ Department of Engineering and Technology Management, Portland State University, Portland, OR 97207, USA
}

\begin{abstract}
Due to a lack of available data, many early planning decisions in Engineering and Technology Management have to be based on experts' opinions and their qualitative statements about evolving technologies, markets and general business environments. Several authors have suggested the use of Fuzzy Cognitive Maps (FCMs) to analytically support these decisions with simulation models that can cope with qualitative information. However, only little practice experience is documented. Based on multiple case studies and an extensive literature review, the paper reviews the state-of-the-art of FCMpractice and introduces a six-step guideline for practitioners and researchers who wish to apply FCMs to real-world problems.
\end{abstract}

\section{INTRODUCTION}

Fuzzy Cognitive Maps (FCMs) - a modeling approach that makes qualitative cause maps computable - have been suggested for decision-support in complex planning situations, such as the planning of information systems [11], the planning of user interfaces [19] and data exchange formats [15] in product development, and the reengineering of business processes [25]. They have also been covered by several PICMET papers that suggested the use of FCMs for technology selection [21] and for product planning in the fuzzy front-end of product development [7].

However, most of these publications remain on a theoretical level: they discuss extensions and modifications to the existing FCM modeling approaches and algorithms, as well as possible application areas, but very rarely contain other than illustrative example cases. Rigid tests of FCM modeling "in the field" are still not available. Also, there are no practical guidelines for FCM modeling, validation and use. Consequently, researchers and practitioners who are interested in FCM application are forced to come up with solutions of their own, rather than focusing on implementation and field test. The following paper attempts to close the FCM implementation gap and introduces a sixstep process model for FCM-modeling that was derived from an extensive literature survey and a series of exploratory case studies.

Following this introduction, the paper is organized in nine sections. Section II provides a short introduction to FCMs and discusses their potentials for decision-support in engineering and technology management. Section III briefly describes the state-of-the-art of FCM practice, relevant findings in related disciplines, and a series of case studies on FCM modeling that have resulted in a guideline for FCM modeling for researchers and practitioners. The modeling steps according to the guideline, its key recommendations and the research that has let to its formulation are presented in section IV (Analysis of objectives and information needs), section V (Analysis of means to fulfill information needs), VI (Knowledge Capture), VII (Conceptual and detailed design of FCM models), and VIII (Test and Validation). Section IX discusses the findings and concludes the paper.

\section{FUZZY COGNITIVE MAPPING}

Fuzzy Cognitive Maps are based on causal maps (sometimes also referred to as cause maps, influence diagrams, oval maps and cognitive maps), that show so-called "concepts" - the elements or variables of a complex system - and the causal links between them. Concepts and causal links are represented as the nodes and arrows of a digraph (see Fig. 1). A positive (negative) arrow between concept A and $B$ means that A causally increases (decreases) B [6].

Cause maps are used to capture experts causal knowledge on complex matters, such as foreign policy, competitor analysis, pricing decisions, and business scenario development [e.g. 1, 4]. The graphic nature of cause maps and their relative simplicity have turned them into an accepted tool for visualization and communication, e.g. in strategy workshops. Their relevance for more far reaching decision support, however, is questionable: Causal maps encode dynamic behavior ("something happens because and after something else has happened"), but the dynamic properties of the mapped system cannot be easily inferred by decision makers. Cognitive limitations prevent them from assessing dynamic system states, especially when feedback loops occur or concepts are embedded in a long chain of causal links [8, pp 225-244]. In order to calculate the cumulated effects of several causal paths on one concept, Axelrod has therefore suggested simple path operations that are based on the path-wise multiplication and addition of signs. The so-called "indeterminacy problem", however, prevails: positive and negative incoming arrows (partially or totally) compensate each other and some concept changes can therefore not be determined [1, pp 63].

Kosko targets the problem of inferring dynamic behavior from cause maps through the use of fuzzy sets and artificial neural networks $[12,13]$.

\section{A. Fuzzy Set Theory}

Fuzzy Set Theory (FST) is an extension to the classic theory of sets (for an introduction and overview, see [26]). In classic set theory, an object is either member of a specific set or not: a person is e.g. either member of the "set of smokers" or member of the "set of non-smokers". Fuzzy Sets are "fuzzy" in the sense that their elements have different degrees of membership to the set. If someone smokes one cigar a year on his birthday, his degree of membership to the fuzzy set of 
non-smokers is very high and his degree of membership to the fuzzy set of smokers is very small, but he is nevertheless member of both sets. The degree of membership of a set of objects X (e.g. people who smoke) to the Fuzzy Set $\tilde{\mathrm{A}}$ on $\mathrm{X}$ is determined by a membership function

$$
\mu_{\breve{A}}: X \rightarrow R
$$

that assigns a real value (usually in the interval $[0 ; 1]$ ) to all elements $\mathrm{x}$, which expresses their degree of membership to $\tilde{\mathrm{A}}$. $\tilde{\mathrm{A}}$ is thus defined as follows:

$$
\widetilde{A}:=\left\{\left(x, \mu_{\widetilde{A}}(x)\right), x \in X\right\}
$$

Its fuzzy complement is defined as:

$$
\mu_{\widetilde{A}^{\mathrm{c}}}(x)=1-\mu_{\widetilde{A}}(x) \forall x \in X
$$

Kosko interprets causal influence in a cognitive map in terms of varying degrees of membership [12]. Physicians e.g. explore the causes of lung cancer by building sub-sets and investigating how many members of the set of people with lung cancer are also member of the set of smokers, of the set of people exhibited to hazardous materials, and of the set of people with cases of cancer in their family. When people with a high degree of membership in the lung cancer set regularly also have a high degree of membership in the subset of smokers, this is interpreted as "smoking causes lung cancer". The strength of causal links can hereby vary. The overlap between the fuzzy set of lung cancer patients and the fuzzy subset of smokers is, for example, much bigger than the overlap between the lung cancer set and the fuzzy subset of people with cancer in their families: smoking strongly causes lung cancer, while heritage only has a weak causal link.

This understanding of causality offers a theoretical framework for the interpretation of cause maps that has some distinct advantages:

- Many concepts in social science and business, such as equality, justice, customer satisfaction etc. are per se "fuzzy" - rather than trying to turn them into crisp concepts (e.g. "satisfied customers rate our company with 3.5 or above") this fuzziness is expressed.

- When people are asked for the views, their responses are often subject to linguistic imprecision. Respondents e.g. consider causal influences to be "very strong", "only minor", or "not very strong". Fuzzy Sets are a powerful means to represent these views.

- Since all fuzzy sets can be represented by their complement, incoming arrows with different signs can be avoided and the indeterminacy problem is solved. Axelrod's path operations are hereby translated into their "fuzzy" equivalent - intersection and union with minimum and maximum operator [12].

There are some drawbacks, however. Representing concepts through fuzzy sets and their complements doubles the number of concepts and thus increases cognitive complexity and data processing demands. Furthermore it leads to awkward concepts, such as "Non-transaction Costs".
In practice, this approach is therefore rarely used [8, pp. 253254].

Dealing with Fuzzy Sets in causal maps furthermore raises additional questions, among others the choice of set operations (operations in classic set theory, such as union and intersection can be generalized to fuzzy sets in more than one way) and the need to "defuzzify" the fuzzy results that is meaningful to the decision-makers. In the overwhelming part of FCM literature (also in Kosko's later publications) FCMs are therefore not based on fuzzy sets but on crisp values: Concepts in FCMs may take values in the range $[-1 ; 1]$ or $[0 ; 1]$. The strength auf causal links is typically expressed by values in the range $[-1 ; 1]$. The idea of "fuzziness" nevertheless prevails, because concepts are not either inactive $(-1$ or 0$)$ or active (1) but take (crisp) values in between. Also the crisp values for causal links are often acquired through qualitative scales that translate statements like "strong to very strong causality" into values like e.g. 0.8. In practice, FCMs are consequently often applied without any reference to FST. Kosko's second extension of traditional cause maps - the use of neural network theory - however, is highly relevant for FCM application.

\section{B. Neural Network Theory}

FCMs consist of simple rules, such as "if it rains, the water level rises". The rules are not hierarchically organized, but part of a network. When one rule becomes effective, this causes other rules to come in effect, which cause yet another set of rules to come in force. As a result, it can be difficult to determine which of the many rules of a complex system are effective at a given time and which one will remain in force on the long run. To solve this problem, Kosko applies Neural Network Theory to FCMs $[13,14]$ He interprets FCMs as recursive neural networks, with concepts being the "neurons" and arrows being the "connections" of the neural network.

Neurons are primitive information processors that take up and aggregate input signals and turn them into an output signal that is sent to other neurons. When the signal is strong enough, the receiving neurons also send output signals ("fire"), thus activating additional neurons or reactivating the ones that have already fired. Thus, activation of one neuron can spread through the entire network.

Three things happen in the neurons of (simple) artificial neural networks: (1) the input signals of the "firing" neurons are taken in, weighted with the connection strengths of the neurons and added, (2) the resulting value is entered into an activation function that determines the state of activation of the neuron, and (3) the activation is entered into an output function that delivers the output values of the neuron.

Kosko takes the same approach to FCMs. Causal links (e.g. the arrow between $\mathrm{C} 1$ and $\mathrm{C} 2$ in Fig. 1) are the connections. They can be represented in an adjacency (or weight) matrix (Fig. 1). Negative causality is hereby usually represented through -1 , positive causality through 1 , and 0 is assigned to edges were no causal relations exist [13]. 


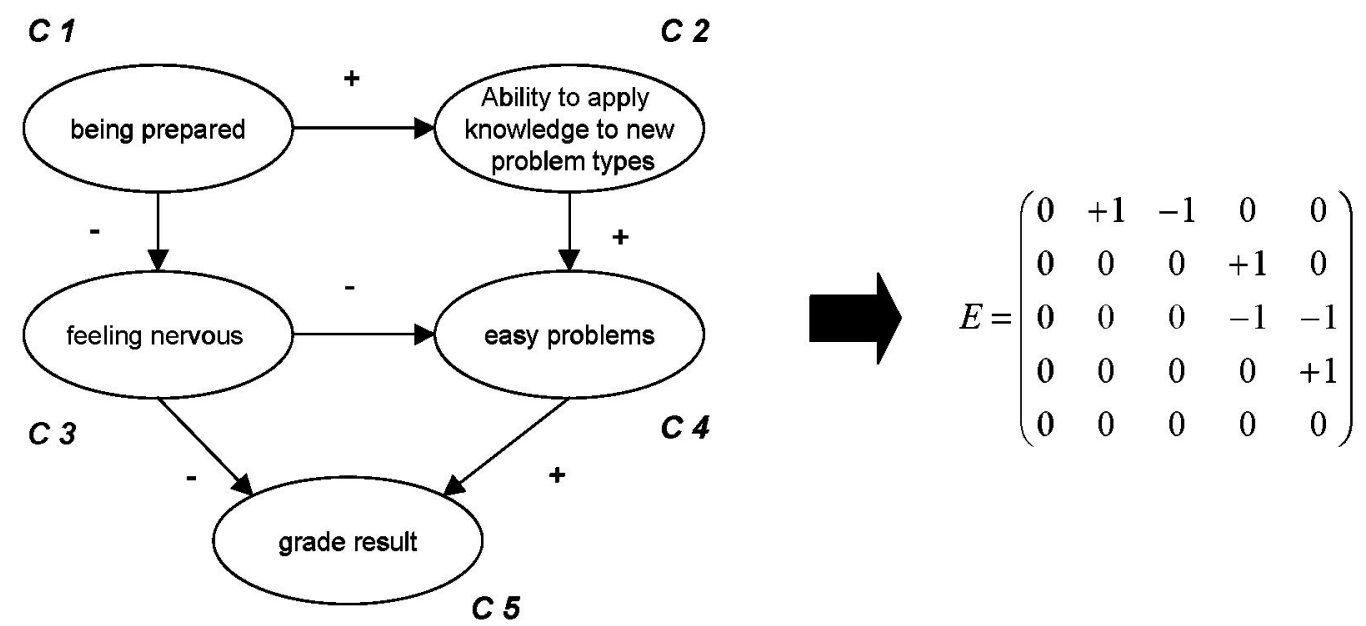

Fig.1: Causal map and adjacency matrix of a student, showing the causal influences on his grade result

The concepts of FCMs (e.g. "C5 - good grade") are neurons - non-linear functions that transform the weighted inputs into outputs in $[0 ; 1]$. Their initial state (the input signals) is represented by a state vector - if, for example, the student in Figure 1 is well prepared. $\mathrm{C}_{1}$ is active (assigned value: 1), while all other concepts are inactive (assigned values: 0$)$. The state vector therefore is $\left(\begin{array}{lllll}1 & 0 & 0 & 0 & 0\end{array}\right) .{ }^{1}$

To calculate the activation level of all concepts, the state vector is multiplied with the adjacency matrix. The result is a new state vector: $S=I_{1} \times E=\left(\begin{array}{lllll}0 & 1 & -1 & 0 & 0\end{array}\right)$

A non-linear output function translates the resulting activation levels into the neurons' output values. Kosko suggests the use of a simple binary function, such as:

$$
\begin{aligned}
& S_{i}\left(x_{i}\right)=0 \text { for } x_{i} \leq 0 \\
& S_{i}\left(x_{i}\right)=1 \text { for } x_{i}>0
\end{aligned}
$$

The resulting output vector is $S_{1}=\left(\begin{array}{lllll}0 & 1 & 0 & 0 & 0\end{array}\right)$

However, since the student remains prepared in the following cycles, the output vector has to be modified by clamping $\mathrm{C}_{1}$.

$$
I_{2}=\left(\begin{array}{lllll}
1 & 1 & 0 & 0 & 0
\end{array}\right)
$$

This vector is again multiplied with the adjacency matrix and entered into the output function, leading to a new output vector. This is repeated until a stop-criterion is met or until the system settles down and new matrix multiplications always lead to the same state vector.

In the case of the student's FCM, the system settles down after the fourth multiplication. The last input vector is:

$$
I_{4}=\left(\begin{array}{lllll}
1 & 1 & 0 & 1 & 1
\end{array}\right)
$$

Kosko calls FCMs of this type with bivalent nodes $(1 ; 0)$ and trivalent edges $(-1 ; 0 ; 1)$ "simple FCMs". They are easy to

\footnotetext{
${ }^{1}$ If the student was well-prepared and nervous, the state vector would be ( 10 $100)$.
}

calculate and modelers only have to decide about the direction of causality and not about the weights of causal links - usually respondents are very confident in doing this. Because of their simplicity, they are quite popular in literature. However, since they are not really "fuzzy", they can be inadequate for problems where concept states are "neither black nor white" but can take "shades of gray". In these cases sigmoid output functions are used, which activate concepts in varying degrees in $[0 ; 1]$ or $[-1 ; 1][8$, pp 273283].

FCMs' system behavior does not only depend on the structure of the underlying causal map, resulting adjacency matrix, and the choice of the start vector (including the question if concept states are clamped or not), but to a large extend on the choice of activation and output functions for every single concept. As a result, there are only few general statements possible about FCMs dynamic behavior:

- FCMs have "meta-rules", which means that several input vectors - so-called input regions -lead to the same final system state. The meat-rules of a FCM can be identified experimentally through simulation [2] and, if strict restrictions are met, analytically [16].

- FCMs with bi- or trivalent concept states (so-called "finite state machines") have meta-rules that stabilize the system in a fixed point or a limit cycle after a few iteration. This means that reentering the output vector into the system does not lead to a different output vector or, alternatively, activates a cycle of vectors that finally results in the same final state. In "continuous state machines" - FCMs with concept values in the intervals $[0 ; 1]$ or $[-1 ; 1]-$ chaotic system behavior is possible, though it seems to occur extremely rare in real-world applications that are characterized by relatively small models with few interdependencies [8, pp. 277-280; 24]. 


\section{Potentials of FCMs in Engineering and Technology Management}

Many real-world decision problems in Engineering and Technology Management, such as forecasting a stillevolving technology, planning a company's R\&D portfolio, or assessing different product concepts in alternative business scenarios, are ill-structured. Decision-makers therefore first of all need to "make sense" of the problem by identifying important system variables, their impact on the desired target variables and the underlying system dynamics. Furthermore most problems are too complex for one decision-maker alone, but can only be solved when the expertise of multiple people is taken into account. FCM-modeling facilitates sensemaking based on multiple experts in several ways $[2,8,13$, 14]:

- FCMs document expert knowledge in networks of simple causal rules, using every-day language. Knowledge acquisition is therefore relatively simple. Since no rule hierarchies need to be defined, FCMs are easily extendable and thus accommodate a sequential modeling approach in which multiple experts add their knowledge to an existing model.

- FCMs are a means to document and communicate experts' mental models of the decision problem through causal maps. They thus make individual "worldviews" explicit and testable. This not only improves experts' individual problem perception, but can also foster a common understanding of the problem in decision-teams.

- FCMs make qualitative causal maps computable and thus assessable for simulation. This enables decision makers to identify critical system elements that they should focus on when searching for decision alternatives. FCM-simulations can furthermore be used to experiment with different decision alternatives and compare their outcome holistically, i.e. with regard to all variables of interest Thus, complex decision problems can be dealt with.

- FCMs can handle qualitative concepts and imprecise causal links and are thus ideal for early planning stages, when little or no reliable quantitative information is available.

Because of these characteristics, FCMs are an interesting, potentially powerful support technology for decision-making in Engineering and Technology Management and a variety of authors have suggested their use [e.g. 7,21$]$. However, the great theoretical potential of FCMs can only be translated into real-world solutions through good modeling practice, which will be discussed in the following sections.

\section{CURRENT FCM PRACTICE}

Because of their roots in qualitatively oriented social science and technical disciplines such as neural network theory, FCMs appeal to researchers with different backgrounds. Consequently, FCM applications vary and a unifying "State-of-the-Art" for FCM practice does not exist, as the variety of FCM topics, the limited scope of the individual research and the different platforms for publication in Table I demonstrate.

\begin{tabular}{|c|c|c|c|c|c|c|c|c|c|}
\hline Year & Author(s) & Publication & Topic & (1) & (2) & (3) & (4) & (5) & (6) \\
\hline 1987 & Taber, Siegel & $\begin{array}{l}\text { Conf.Proceedings: } \\
\text { Neural Networks }\end{array}$ & Generating expert weights in combined $\mathrm{FCMs}$ & & & & & & \\
\hline 1989 & Zhang et al. & $\begin{array}{l}\text { Journal: IEEE } \\
\text { Trans. on Systems, } \\
\text { Man \& Cybern. }\end{array}$ & $\begin{array}{l}\text { Extension of traditional FCMs with "negative, } \\
\text { positive, neutral" (NPN)-Logic for more } \\
\text { differentiated modeling }\end{array}$ & & & & & & \\
\hline 1991 & Taber & $\begin{array}{l}\text { Journal: Expert } \\
\text { Systems with } \\
\text { Applications. }\end{array}$ & $\begin{array}{l}\text { Knowledge Processing through FCMs; FCMs } \\
\text { based on the combined knowledge of multiple } \\
\text { experts }\end{array}$ & & & & & & \\
\hline 1994 & Craiger, Coovert & $\begin{array}{l}\text { Conf. Proceed.: } \\
\text { Intern. Conf. on } \\
\text { Fuzzy Systems }\end{array}$ & $\begin{array}{l}\text { FCM-Modeling of dynamic social and } \\
\text { psychological problems, illustrated with example: } \\
\text { "family problems" }\end{array}$ & & & & & & \\
\hline 1994 & Taber & Journal: AI Expert & $\begin{array}{l}\text { Presentation of FCM Method; Modeling of social } \\
\text { systems, illustrated with example 'Reform of the } \\
\text { health care system" }\end{array}$ & & & & & & \\
\hline 1995 & Park, Kim & $\begin{array}{l}\text { Journal: Int. J. } \\
\text { Human-Computer } \\
\text { Studies }\end{array}$ & $\begin{array}{l}\text { Extension of traditional FCMs to represent time } \\
\text { relationships }\end{array}$ & & & & & & \\
\hline 1997 & Bryson et al. & $\begin{array}{l}\text { Conf. Proceed: } \\
\text { Intell. Information } \\
\text { Systems }\end{array}$ & $\begin{array}{l}\text { FCM based on the combined knowledge of } \\
\text { multiple experts. }\end{array}$ & & & & & & \\
\hline 1998 & Lee et al. & Journal: Simulation & Strategic Planning based on FCM-Modeling & & & & & & \\
\hline 1998 & $\begin{array}{l}\text { Stylios, } \\
\text { Groumpos }\end{array}$ & $\begin{array}{l}\text { Journal: Journal of } \\
\text { Intelligent } \\
\text { Manufacturing }\end{array}$ & $\begin{array}{l}\text { Modeling of a process control for a production } \\
\text { process }\end{array}$ & & & & & & \\
\hline 1998 & Schneider et al. & $\begin{array}{l}\text { Journal: Fuzzy Sets } \\
\text { and Systems }\end{array}$ & Automated construction of FCMs & & & & & & \\
\hline
\end{tabular}


TABLE I. STATE-OF-THE-ART OF FCM PRACTICE (cont.)

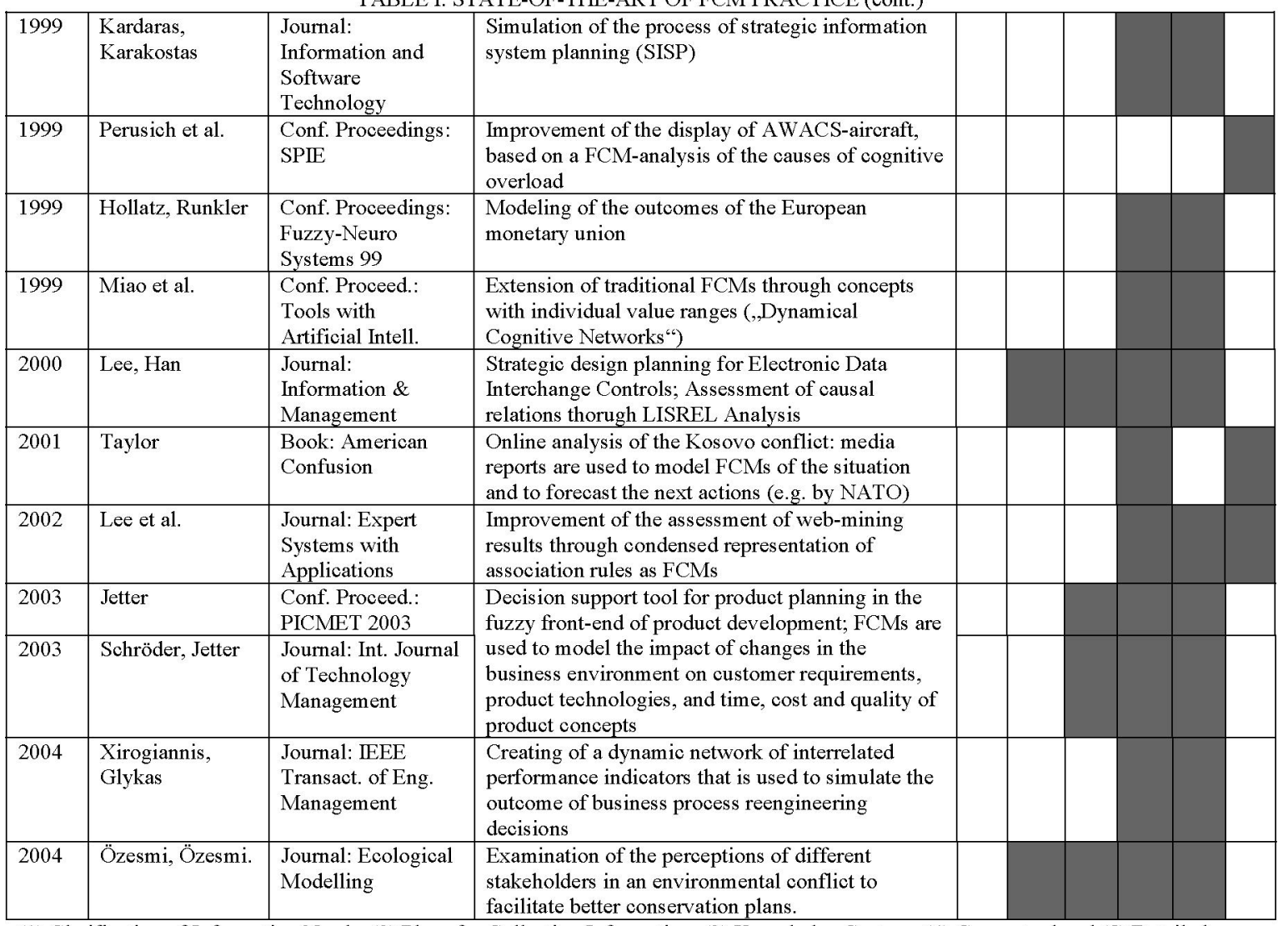

(1) Clarification of Information Needs, (2) Plans for Collecting Information, (3) Knowledge Capture, (4) Conceptual and (5) Detailed

Design, incl. model usage, (6) Test, Interpretation, Validation;

highlighted areas $=$ focus of the research

This causes a considerable problem for researchers and practitioners, who want to use FCMs to solve real-world problems in engineering and technology management: rather than solely focusing on the application, test and critical reflection on FCMs, they have to overcome a large number of practical questions that are only poorly addressed in academic literature.

When attempting to implement and test a FCM-based product planning tool first introduced on PICMET 2003 [7] we encountered the same difficulties and knew little about the many transformation steps required to turn expert knowledge into a usable and tested FCM model. As can be inferred from Table 1, some of the required activities are rarely addressed in publications on FCMs, mainly because many writings theoretically extend, rather than to practically apply FCMs. Consequently, little is said about how experts can be identified, and how their knowledge can be elicited and translated into FCM-models. Instead, researchers simply build models based on their individual understanding of a problem without involving outside experts. Also very little is written on how models should be tested and how FCMresults can and should be interpreted.

In order to close this "practice gap", we searched for answers outside the scope of typical FCM-publications and found a wealth of information in different disciplines: Clarification of information needs and identification of experts (and/or stakeholders), for example, are common problems for the designers of expert systems [5], as well as for business strategists. Cognitive psychologist employ a variety of methods (e.g. interview techniques and mapping exercises) to capturer knowledge contents (the meaning of concepts) and knowledge structures (their causal relations) that can be applied to FCM modeling $[9,10]$ while model test and validation play a prominent role in the application of System Dynamics [23].

In addition to a broad literature survey, we investigated several aspects of FCM practice in a series of exploratory case studies (see references for details):

- Case Study 1: A case study with two groups of students (total of 13 undergraduates and graduates from various disciplines, mainly engineering and business), in which the students drew causal maps without any personalized interaction with a knowledge engineer or interviewer. The cognitive mapping process and the resulting causal maps were analyzed with regard to content and structure, possible problems and defects, and usability for FCM modeling [8, pp. 313-339]. 
- Case Study 2: In a second case study, one of the two groups of students furthermore took part in a moderated team discussion during which a causal map was developed jointly and with the help of a researcher. The resulting map was compared to a map that was automatically constructed from the individual maps of the test persons [8, pp. 340348].

- Case Study 3: A third case study assessed the potential of a simple text-mining tool for identifying concepts that are relevant for a knowledge domain and should be incorporated in FCMs [8, pp. 348-353].

- Case Study 4: In a fourth case study, the application of FCMs in the fuzzy front-end of product development, as suggested in $[7,20]$ was put to test in the product planning stage for a new laser machine in a small high-tech company [8, pp. $377-435]$.

Furthermore experience with different interview techniques (open, unstructured interview; episodic interview; free word association) and different mapping formats (ontologies for concept meanings; process maps) was gathered through two case studies among SME practitioners $[9,10]$. In the following sections, the findings of these research efforts are summarized and described, using a sixstep process model [ 8 pp 354-364] that is visualized in Fig. 2:

- Clarification of objectives and information needs (Step 1).

This includes the definition of the scope of the modeling project (topic, model boundaries, timeframe under consideration, etc.), as well as a list of relevant questions about the knowledge domain.

- Plans for collecting relevant information (Step 2). This modeling steps requires the identification of knowledgeable informers (experts, stakeholders, publications, etc.), as well as planning the knowledge elicitation techniques (interviews, text analysis, mapping exercises, etc.) to be used.

- Knowledge capture (Step 3). This process step includes all activities that lead to (weighted) causal maps about the knowledge domain, as well as to information about the expected dynamic behavior of the system they represent.

- Conceptual (Step 4) and detailed (Step 5) design of the FCM model, including choice of squashing functions and input vectors and consideration of time lags.

- Test, Interpretation, and Validation of model results (Step 6).

As indicated by the "star shape" in Fig. 2, this six-step process is not purely sequential: An expert identified in step 2 , for example, could turn out to be less knowledgeable than anticipated in step 3 , thus necessitating a new information analysis. The expert might also point out, that the model objectives - the question to be answered - are too broad or too narrow, thus forcing the modeler to go back to step 1 . Furthermore, a model could fail the practice test in step 6 because it is poorly parameterized (return to step 5), conceptually problematic (return to step 4) or because it does not answer the right questions (return to step 1)

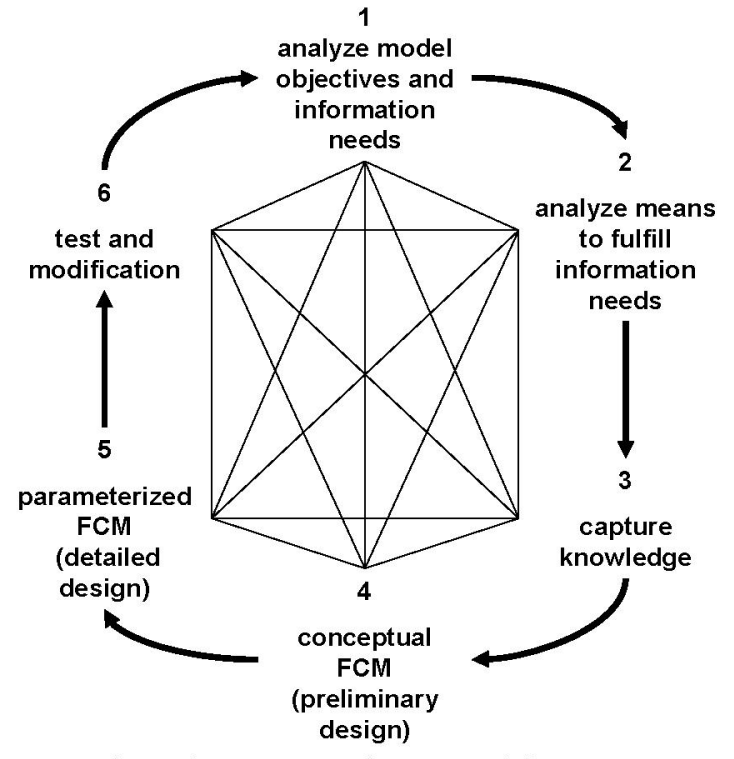

Fig. 2: Six process steps for FCM modeling [8]

The six-step process model is a heuristic approach that cannot guarantee optimal results. However, if employed rigorously it can make sure that every transformation step (e.g. the transformation from knowledge in the expert's head into a causal map) is verified, thus ensuring the quality of the FCM-modeling outcome.

\section{ANALYSIS OF OBJECTIVES AND INFORMATION NEEDS (STEP 1)}

In case study 1 , test persons were asked to explain the causes for right-wing extremism among youth. A surprisingly large number of them (4 out of 13) came up with causal maps (some of them relatively large and elaborate) that did not contain the concept of "right-wing extremism" or any remotely similar concept $\left[\begin{array}{lll}8 & \mathrm{p} & 326\end{array}\right]$. The test persons obviously lacked a clear problem focus. To avoid this problem, every modeling project must start with a thorough analysis of the model's objectives - ,the single most important ingredient of a successful modeling study “ [23, p. 89].

Unspecific mission statements (e.g. "model, how our market will evolve in the future" or "show everything that affects our sales") can lead to large, overly complex "world models" that break the rule poignantly stated by Sterman: "Always model a problem. Never model a system" [23, p. 90]. In order to clarify the objectives of the modeling project at hand, modelers should inquire about problems and undesired states that should be changed as well as about alternatives decision-makers can choose from in the given situation [8, pp 355-356].

Thorough inquiry not only ensures the problemorientation of the model, but also helps to identify target variables of the model (e.g. "sales", "market share", or "savings in manufacturing costs") that can be used in the probing phase of knowledge elicitation, as well as 
stakeholders whose views and knowledge might be important for the model [8, pp 355-356].

Once the model objectives are known, they should be documented in a one-sentence mission statement. Model boundaries - the variables that should be excluded from the model or considered to be exogenous - should be assessed and documented in a model boundary chart. Also, any questions and information needs that become apparent during the goal analysis should be documented, preferably as question. Finally, the timeframe (present situation, developments in the next 2 years, situation in 10 years, etc.) of the analysis needs to be clarified [8, pp 355-356].

\section{ANALYSIS OF MEANS TO FULFILL INFORMATION NEEDS (STEP 2)}

In order to fulfill the information need, modelers have to decide on the people ("experts") whose domain knowledge should be used for the model and on the means (interviews, text analysis, etc.) to access this knowledge and to translate it in a (possibly still crude) causal map that is the basis for FCM modeling. Both decisions are closely intertwined because in many cases the choice of experts also determines the way in which expert knowledge is captured.

\section{A. Identifying Experts}

In the strict sense of the word, "experts" are people with substantially more experience than average in a relatively small field of expertise, such as fault analysis in a technical system. Other than less experienced people, experts only need little information to analyze a problem and to choose the matching solution from the cases they have accumulated in their memories. Problem-solving is therefore almost automatic and often experts are not even fully aware of how they have solved a particular problem [5]. These "real" experts are usually well-known in an organization and are therefore relatively easy to identify.

For many broad knowledge domains, such as the reasons for right wing extremism or the influencing factors on future customer requirements, this type of expertise does not exist. However, there are usually many people who have some knowledge in some aspect of the knowledge domain. They are a good starting point for identifying additional "partial experts" by means of snowball sampling. One can, for example, ask them to give five names of people that they regularly consult and/or consider experts in a particular field. By comparing the names on different people's lists, experts often become obvious. This approach can be systemized, using organizational network analysis [6].

A second approach to finding experts in broad knowledge domains is stakeholder analysis. Customers, e.g., are clearly stakeholder in future vehicle designs, but so are environmental groups, law-makers, suppliers, etc. By collecting information from people who belong to the different groups, one can get a broad and fairly complete picture of the knowledge domain in question.
When a potential expert is identified, his or her objectivity, ability and motivation to share knowledge have to be carefully considered:

- Objectivity, though certainly claimed by most experts, can be a problem because experts have individual beliefs, values, and "worldviews" that might not be entirely objective in a scientific sense. It is therefore important to choose experts who at least attempt to give an unbiased account of their knowledge and who do not misuse the modeling project to follow their own agenda. Also, models should never exclusively be based on only one or a few experts' opinion but existing theoretical knowledge and empirical evidence needs to be incorporated in the model [3].

- Ability can be a problem when language barriers exist, when experts extensively use technical jargon and when organizational problems (distance, lack of time) occur. Lack of ability, furthermore, might occur with "real" experts with automated problem-solving who sometimes cannot fully articulate the approach that they use [5].

- Lack of motivation is highly problematic, when experts fear negative consequences of sharing their knowledge, such as conflict with peers or the loss of prestige and power when they are not the only experts any more. To be motivated, experts furthermore need to benefit from sharing knowledge, e.g., through recognition, through the satisfaction of being considered an expert or through future ease of work. If a poorly motivated expert takes part in a modeling project, he might not give a lot of thoughts to his answers or - even worse - deliberately distorts results [5]

As a consequence, not everybody who has relevant knowledge is a suitable partner for knowledge elicitation sometimes the expert with the greatest knowledge is not the top choice but a more accessible, better motivated or less specialized person should be asked.

Finally it is important to note that even the most carefully selected and most experienced expert might be "wrong" in the sense that his mental model of the problem is inadequate for its solution. Testing and comparing model results with real-world feedback is therefore mandatory and will be further discussed in Section VIII.

\section{$B$ Deciding on Methods for Knowledge Capture}

Once the experts for a knowledge domain have been identified, knowledge capture has to be planned. In principal, four alternative approaches exist (see Table II) [8,pp.262]:

TABLE II. ALTERNATIVE APPROACHES TO KNOWLEDGE

$$
\text { CAPTURE }
$$

\begin{tabular}{|c|c|c|c|}
\hline & $\begin{array}{l}\text { the modeler is } \\
\text { the expert }\end{array}$ & $\begin{array}{l}\text { the modeler } \\
\text { interviews } \\
\text { the expert }\end{array}$ & $\begin{array}{l}\text { the modeler } \\
\text { analyzes } \\
\text { documents }\end{array}$ \\
\hline $\begin{array}{l}\text { causal knowledge of } \\
\text { one expert }\end{array}$ & Option 1 & \multirow{2}{*}{ Option 2} & \multirow{2}{*}{ Option 3} \\
\hline $\begin{array}{l}\text { causal knowledge of } \\
\text { a group of experts }\end{array}$ & & & \\
\hline $\begin{array}{l}\text { combination of the } \\
\text { causal knowledge of } \\
\text { different experts }\end{array}$ & & \multicolumn{2}{|l|}{ Option 4} \\
\hline
\end{tabular}


1) Option 1 - The modeler is the expert: When the modeler is knowledgeable about the domain that is to be represented, he can start drawing a cause map and translate it into an FCM right away. He is expert and knowledge engineer in one person. Most publications on FCM deal with this case, because the FCMs described in them are usually used to exemplify extensions of the FCM method, rather than to describe real-world systems. In practice, however, one rarely finds experts that are experienced FCM-modelers and who are knowledgeable enough that reliable models can be built exclusively based on their views.

2) Option 2 - The modeler interviews the expert(s): Interviews are powerful means to focus the experts' attention on a particular knowledge domain and to capture their knowledge in depth. Interview techniques are applied in various disciplines, such as expert system design, social science, and psychology. They can take different forms, from informal, unstructured or only semi-structured conversations with individual experts to highly structured group meetings or closed question questionnaires [10]. The advantage of interviews is that they can be focused on the information needs identified in step 1 and thus deliver relevant and usable results. Furthermore, a well laid-out interview can make sure that the experts' knowledge is correctly reflected in the causal map and the resulting FCM model. A potential disadvantage is the interview situation, which might distract experts or prevent them from sharing all their views and ideas. Furthermore, interviews - especially when they take place facet-to-face and are not administered by a closed-question questionnaire - tend to be time consuming. Not only do the experts and the knowledge-engineer have to meet, but the interview transcripts also have to be processed and translated into an FCM model (see [17] for a good explanation on how to transfer interview transcripts into causal maps).
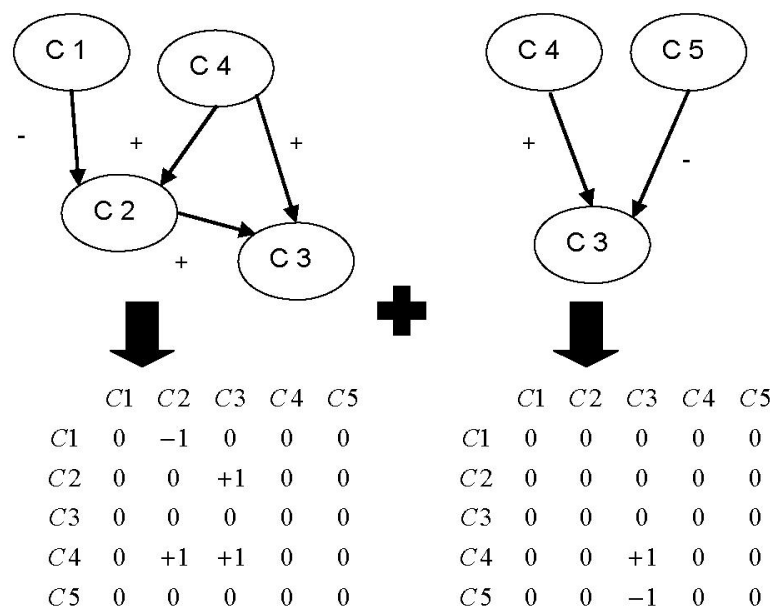

Fig. 3-Combination of FCMs
3) Option 3 - The modeler analyzes documents: When the knowledge domain in question is well-documented in publications, modelers can read text documents and infer causal maps from them. To be useful, documents do not necessarily have to be written with the intention to present a knowledge domain, such as textbooks or scientific publications. Relevant knowledge can also be "hidden" in industry trend reports, market studies, newspaper articles, interviews (e.g. on TV), press statements, annual reports, etc. This type of documents can be particularly useful to model aspects for which expertise resides in more than one person and is therefore expensive to access (e.g. knowledge about global industry trends) or in cases where experts are not willing to take part in a modeling project (e.g. competitor strategies).

4) Option 4-Combination of causal knowledge of different experts: For many knowledge domains, multiple knowledge sources have to be integrated. Integration can take place through one person, who studies all knowledge sources, thus becomes an expert in the field and translates the so acquired knowledge into a causal map (see Option 1). Alternatively, knowledge can be integrated in meetings and group discussions of the experts (see Option 2). This is, however, infeasible and inefficient, when real expertise does not exist, but a large number of sources are "somewhat knowledgeable" and when each of these sources contributes only little to the understanding of the domain. The added value of each source simply does not justify its presence in a group discussion. In these cases, it can be useful to first translate the experts' knowledge into individual causal maps, express them as adjacency matrices of the same size and combine them ex-post, using an approach suggested by Kosko [14] (see Fig. 3). To make allowance for different levels of expertise, credibility weights can be assigned to experts.

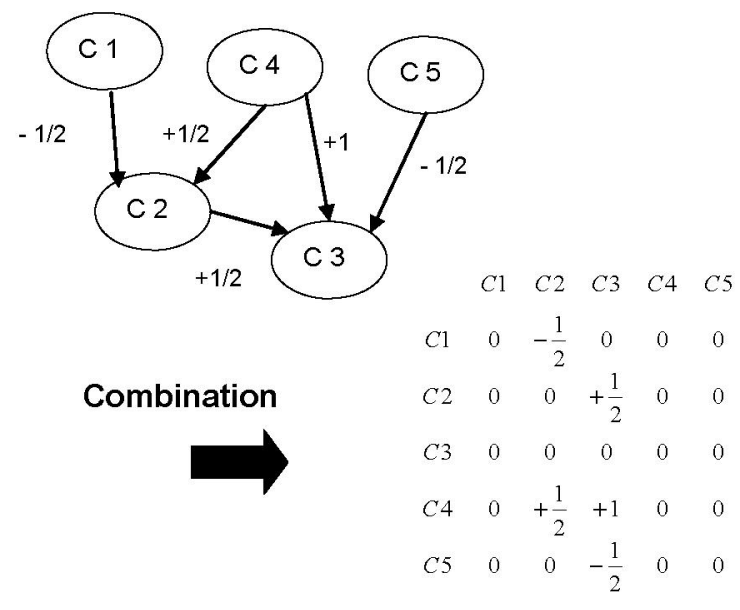




\section{KNOWLEDGE CAPTURE (STEP 3)}

\section{A Cognitive Mapping}

Once experts are identified and the methods for knowledge capture are decided upon, the experts' knowledge about the domain has to be elicited and transferred into a causal map. This can be achieved through cognitive mapping - a process in which the experts themselves document their understanding of a knowledge domain in (causal) "cognitive maps". This approach is theoretically convineing: The method is easy to learn and intuitive (in case study 1 , total training time was about 20 minutes) $[8$ p. 315]. It is not obtrusive and thus reduces possible distortions of the experts' views and opinions through the interviewer and his translation of the interview results into causal maps. It is said to help experts and decision-makers to become aware of their individual world views and consequently helps them to make better use of their mental models [6]. And finally it is economical: it directly results in causal maps that can be translated into FCMs and it does not require the expert to meet with an interviewer at a specific time and location. Thus, more experts can be involved in building the knowledge base of the modeling project.
While theoretically intriguing, cognitive mapping has a variety of practical problems that are extensively researched and discussed in [8]. The following advice is based on these findings.

\section{1) Separation of content and structure}

Drawing causal maps requires content knowledge about the concepts of the causal map, as well as structural knowledge about the links between the concepts. To reduce the cognitive demands on the expert, content and structure are usually elicited in two separate steps.

In case study 1 [8, pp. 314-138], this was achieved through a sequential approach: the test persons first collected important concepts of the knowledge domain by writing them on note cards (one per card). Secondly they moved the cards on their desk to position them relative to each other in a way that showed causal influence. Thirdly, they drew causal maps ("ovals and arrows") on large pieces of paper. Since pencils and erasers were used, changes could be made at any time. Signs for causal links were added in the fourth step, using the symbols and explanations given in Fig. 4. Causal weights were expressed on 5-point Likert-type scales and were added in the final fifth step.
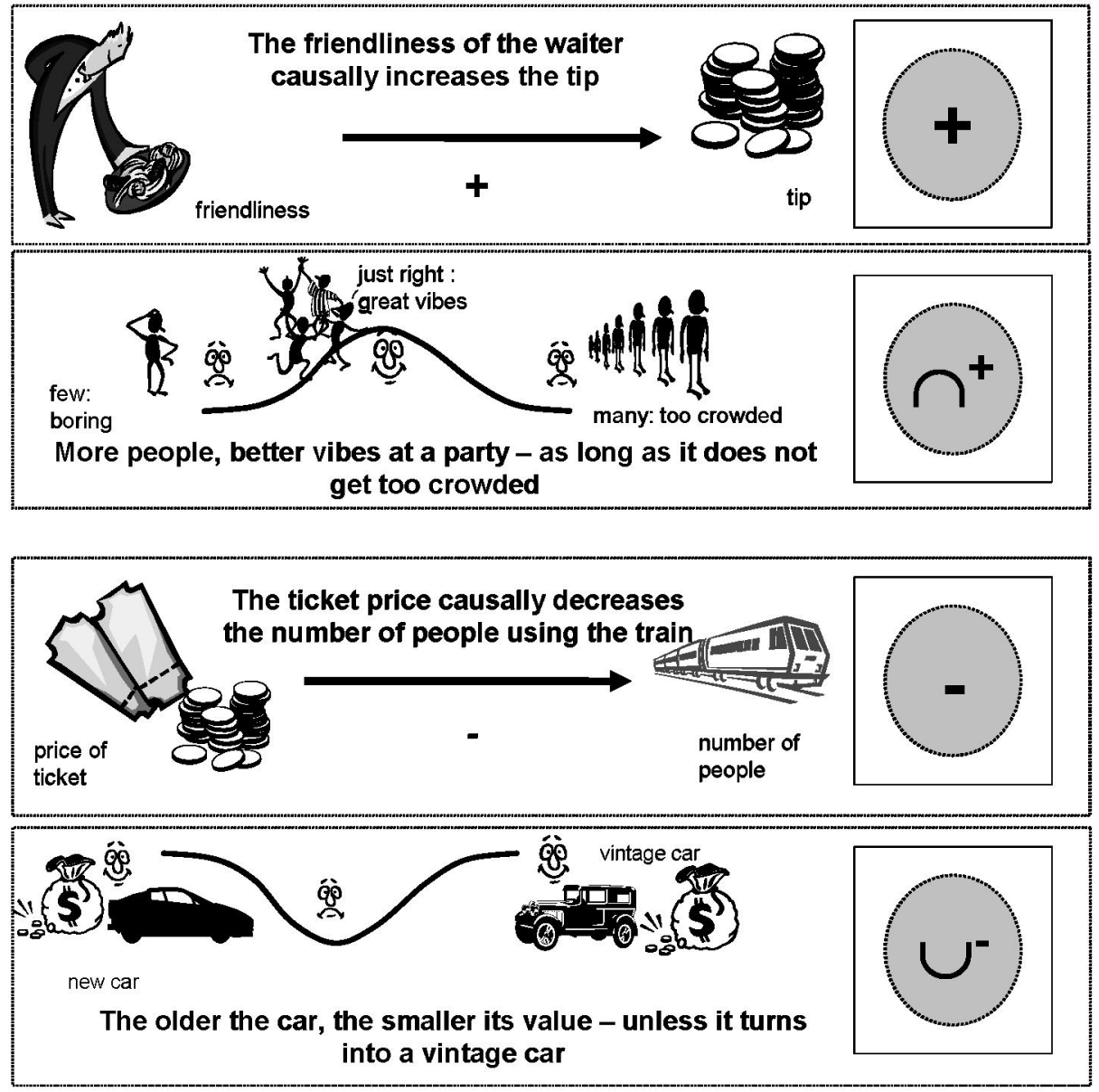

Fig. 4: Instructions and Symbols for Causal Links 
In general, this mapping approach proofed feasible: Respondents were able to complete all mapping steps without intervention of the researchers and expressed satisfaction with the process and their results. The resulting maps contained all necessary information for the subsequent translation into FCM models. We do, however, believe that a more thorough training in causal mapping with some handson exercises could have helped test persons to focus more strongly on their mental models and could have further improved the quality of the map.

The vast majority of respondents only drew positive and linear causal relations, which seems to be the "natural" way of thinking about causality. U-shaped causal links, the use of which is sometimes suggested in literature to capture complex causal links ("Fertilizing the field increases yield until it is too much and over-fertilization damages the soil"), were only very rarely used. We suggest to not use them during causal mapping for two reasons: (1) in the case study, there was some indication that even respondents, who used the symbol, did not fully understand its meaning - it might simply be too demanding for respondents, if they are not very experienced in mapping and modeling. (2) "U-shaped" causality can usually also be expressed in a linear fashion: "Fertilizing the field increases yield, over-fertilizing the field decreases yield."

\section{2) Use of concept cards as stimuli}

Experts can only express knowledge that is available in their short-term memory. Cognitive Mapping, like any other kind of interview technique, should therefore start with the activation of knowledge - its transfer from the long-term memory of the brain, where it is stored, to the short-term memory, where it is processed. Warm-up questions that help the expert to think about the particular knowledge domain are therefore necessary. Activation can furthermore be supported through stimuli, such as note cards with important concepts of the knowledge domain, models, photographs, and role plays [10].

The case studies demonstrated, that note cards with socalled "start concepts" (concepts that every expert's causal map should include, because they are at the core of the knowledge domain and/or represent a critical (target) concept) are useful stimuli for three reasons:

Firstly, they help to standardize the names for key concepts, thus reducing the number of cases in which experts use different terms for the same concept. This standardization is helpful when sharing maps among experts or integrating them into one.

Secondly, the use of "start concepts" reduces the number of cases in which important (target) concepts are entirely missing from the map

Thirdly they introduce the respondents to a useful template for documenting concepts that they should use when adding concepts: concepts that can have different qualities (e.g. good or bad parenting, high and low self-esteem) are expressed as adjective and noun (either "good parenting" or "poor parenting). If this were not the case, two respondents who agree that good parenting increases children's selfesteem and draw the same causal link in their maps ("parenting $\rightarrow$ self-esteem") can still come up with different signs: a positive link (meaning: good parenting causally increases good education) or a negative link (meaning: poor parenting causally decreases self-esteem). This cannot only confuse the respondents but also makes it more difficult to compare and integrate different experts' causal maps.

In case study 3 [8, pp. 348-353], we investigated the potential of a simple statistical approach for identifying domain-specific start concepts that can be used as stimuli during knowledge capture. We assumed that a collection of texts about a specific knowledge domain contains much more domain-specific concepts than every day language, and that the most frequently mentioned concepts in the text collection are highly relevant to the domain and useful as start concepts. To test this assumption, we first created a collection of 149 newspaper articles on a specific knowledge domain ("rightwing extremism among youths"), using an online newspaper archive and a simple two keyword query. We then eliminated words that are very frequently used in every day language (e.g. "and", "or", "the") and therefore common in every document, regardless of the topic, from the text corpus, using so-called stop-lists (1000 and 10000 words). We counted the frequency of the remaining words and focused our analysis on those words that were most frequently used in the "textmining word list". They were useful for defining start concepts for several reasons:

There was a large overlap between the text-mining word list and a list of all concepts mentioned in the experts' causal maps, particularly with regard to important core concepts that were part of many of the experts' maps. However, textmining also delivered several concepts that had not been part of any experts' model but (in retrospect) seem highly relevant to the subject matter. Text-mining for start concepts could be an interesting means to get a broad view on the subject matter, resulting in more complete causal maps.

The approach furthermore delivered a large number of synonyms for general concepts and specified some concepts in greater detail: respondents, for example, used general concepts, such as "right-wing political parties" or "academic experts" while text-mining delivered the actual names of right-wing parties and experts in research institutions. The text-mining approach can thus not only provide hints for the standardization of concepts through a domain-specific thesaurus and can also be used to identify relevant stakeholders and experts.

A drawback of using concept cards as stimuli is a possible distortion of experts' views. They could, for example, integrate start concepts into their causal maps even though they do not really consider them relevant. It is therefore important that respondents do not have the impression that they need to stick to the start concepts provided and that they can add concepts at any time. 


\section{3) Capture of additional, non-causal knowledge}

Cognitive Mapping can result in maps that make perfect sense to the expert but are difficult to comprehend for the FCM modeler. Knowledge capture must therefore not exclusively focus on causal knowledge but must provide clarification of concept meanings and provide information on the expected system behavior.

The meaning of "start concepts" can be easily clarified through a brief description that includes synonyms for the concept. If experts agree with the concept, they use it in their map, if not they come up with a new concept of their own. To provide clarification for these new concepts, an approach adapted from Smithin [22] proofed successful in case study 1 [8, pp. 313-339]: experts were asked to first provide synonyms (or "related words") for their concepts, as well as words that they consider to be in sharp contrast to the concept. Instructions pointed out that the synonyms and antonyms did not have to be "logical" in the way an encyclopedia would require, but should be chosen to illustrate the expert views. In the case study, a respondent, who included "unemployment" in a causal map about causes for extremism, used "lack of things to do" as synonym and "selfactualization" as an antonym, thus indicating that, in his model, he was less interested in the economic situation resulting from unemployment than in the psychological effects. This information can be important for an FCMmodeler who needs to understand causal links drawn in the map. A computer-based causal mapping approach could furthermore also use the respondent's choice of synonyms and antonyms to suggest additional or alternative concepts that better reflect the commonly accepted concept meaning.

Other important background information experts have to provide is their "dynamical hypothesis" [23] about the system's dynamic behavior. They can be used in later stages of the FCM-modeling process to test if the FCM model's system behavior reflects the experts' expectations. Dynamical hypothesis can be captured by asking experts how the system has evolved in the past ("Why do you think this problem has come up?", "How different is $\mathrm{X}$ from what it was a year ago? Why") and how they expect it to change in the future.

\section{4) Capture of Group Knowledge}

In many real-world FCM applications, e.g. when a multidisciplinary team plans a new product, single experts' individual views are not as important as an integrated causal maps that represents the knowledge of all the experts. An important question therefore is how to efficiently capture group knowledge: should the group map be generated in a group discussion, or should it result from the combination of individual causal map, as described as Option 4 (see Fig. 3) above.

In case study 3 [8, pp. 348-353], we investigated this question by comparing a group-generated causal map with a map generated from the individual maps of the group members. The group generated map was substantially smaller (28 concepts) than the combination map (58 concepts). This can be mainly attributed to a standardization of concepts - the group frequently combined similar concepts and integrated it into one. However, there also was an observable loss of content: the group ignored concepts that several group members had included in their individual causal maps. The group map was furthermore only insignificantly denser than the combination map, though a standardization of concepts should have increased density - the group effort obviously did not create additional knowledge about the problem structure. Furthermore, the system behavior of the group map (when modeled as a simple FCM) was less plausible and in line with the group member's dynamic hypothesis than the combination map. Contrary to our expectations, a simple mathematical combination of individual maps thus delivered a more adequate model than the interaction of the experts.

These results, however, should only carefully be generalized, because the group structure and the specifics of the group session (e.g. visualization of the cause map during discussions, available time) certainly have an important influence. Based on these findings, however, it is promising to further investigate a decentralized approach to knowledge capture in which experts provide maps at their time and convenience and do not have to travel to attend group meetings.

\section{5) Evaluation of Cognitive Mapping for Knowledge Capture} Cognitive Mapping, as employed in our cases-studies, is a non-obtrusive, economical way to capture knowledge from multiple experts without extensive involvement of the "bottleneck" of many modeling projects, the knowledge engineer. It is a means to systematically exploit the knowledge capture options 1 and 4 discussed above (see Table II). Efficiency of the process could furthermore be improved through a software solution that is used by the experts to enter individual causal maps and to view other experts' maps, as well as by the modeler to read, edit, and combine expert maps and translate them into FCM models. Such a software could also make sure, that that concepts are clarified (e.g. through an online thesaurus) and standardized, and that entries are checked for completeness and plausibility. With such a quality control in place, the already good quality of experts' causal maps, as demonstrated in the case studies, could probably further be improved, turning Cognitive Mapping into a very attractive approach to capturing knowledge for FCMs in situations when (partial) knowledge resides in many widespread knowledge sources. It should, however, not be used in all situations: When knowledge of a few highly skilled experts needs to be captured in its depth, interview techniques seem more adequate. They are very briefly discussed in the following section.

\section{$B$ Interviews}

Interviews can take place on a one-to-one basis or in the form of group discussions that are moderated by the interviewer or are executed in the style of a Delphi-study. 
Both have advantages and disadvantages: experts might be more willing to give detailed information and voice possibly controversial opinions when they meet with the interviewer in private, rather than having to talk in public in front of a group of peers. On the other hand, well-functioning groups sometimes successfully take up and integrate contrasting views, thus providing a much more coherent and complete picture of the knowledge domain than a series of individual interviews.

The quality of an interview result is not only determined by the expert's knowledge and ability and motivation to share it, but also by the interviewers, who can be responsible for considerable distortions of the expert's views, e.g. by skipping the important knowledge activation and attempting to capture content and structure at the same time, by creating an interview atmosphere that prevents the expert from being open, by misinterpreting the answers, or simply by asking the wrong questions. Every book on social science research methods gives ample advice on how to avoid these problems.

A few aspects, however, are particularly important for capturing knowledge for FCMs [8, pp. 263-265; 10]: Interviewers must carefully plan the interviews to make sure that, even though they focus on causal knowledge, important other knowledge (e.g. about concept meanings) is also captured. They furthermore need to include questions that evoke possibly existing contrasting views (e.g. "Let me play the devil's advocate in response to your story about the need to involve the customer in this decision. What if you did not would this really upset the customer?") and thus help experts to challenge their mental models. They should make the purpose of the interview and the methods (e.g. the use of causal maps as a visualization tool) transparent to the interviewee, thus creating a sense of mutual respect and trust. Finally, any transformation step (e.g. from interview to interview transcript and from transcript to causal map) must be approved by the interviewee as a correct representation of his ideas ("communicative validation")

\section{CONCEPTUAL AND DETAILED DESIGN OF FCM MODELS (STEP 4 AND 5)}

\section{A Refinement of Causal Maps}

The first step of FCM-modeling is refinement of the causal map that was generated during knowledge capture. It is necessary because causal maps, even if generated by an experienced modeler, usually contain concepts and causal links that are adequate for a causal map but cause computational problems for a FCM. The following subsections discuss typical problems [8, pp 326-335]:

\section{1) Disregard for Model Boundaries}

Respondents sometimes include concepts that are excluded in the model boundary chart or link concepts to exogenous variables. These concepts and causal links must be deleted.

\section{2) Definitional or overly detailed causal Links}

Respondents seem to have a tendency to explain those aspects of the knowledge domain that they consider particularly difficult to understand or important in much greater detail than other areas. As a consequence they sometimes draw definitional, rather than causal links (e.g. "new car sale" $\rightarrow$ "total sales" and "used car sales" $\rightarrow$ "total sales") or draw lengthy causal paths. A respondent, who believe that $A$ directly causes $B$, but assumes that this $A$ might not be self-explanatory could, for example, draw a causal path $\mathrm{A}^{* *} \rightarrow \mathrm{A}^{*} \rightarrow \mathrm{A} \rightarrow \mathrm{B}$. If $\mathrm{A}^{* * *}$ "fires", this impulse reaches $B$ with delay and possibly at a point when other influences on B have already weakened. Causal maps therefore have to be checked for possible definitional causal links: when they are strictly definitional they need to be eliminated (e.g. only consider concept "total sales" or "A"). When they are intended to express causal impact on a specific, single concept, they can be organized in a so-called "nested FCM" [2, p. 15] - a sub-model that is calculated separately and delivers an input value for the state vector.

\section{3) Diagnostic Variables}

Respondents sometimes include concepts in their causal maps that have no "Out"-arrows, even though they are clearly not a target concept. This is usually an indicator for an incomplete or faulty knowledge capture. In some cases, however, these concepts are diagnostic variables - they are causally connected to the same concepts that influence the target concepts, but independent from the latter and give information about the state of the system. If chosen wisely (diagnostic variables should reflect readily available, objective information), they can give valuable information for the calibration of the FCM. In case study 1, one respondent e.g. considered right-wing extremism (= target concept) to be part of the same general trend that causes violence among teenagers ( $=$ the diagnostic variable). Both concepts need to change in the same direction if the FCM's dynamic behavior is to correctly reflect the respondent's worldview.

\section{4) Conditional Causality}

Some causal relations are conditional: Concept $\mathrm{A}$ ("Rain") and Concept B ("Temperatures below $0 \mathrm{C}^{\circ}$ ") cause Concept C ("Slippery road") to happen. FCMs need to reflect this either by making sure that the threshold of the activation function of concept $\mathrm{C}$ can only be met by $\mathrm{A}$ and $\mathrm{B}$ together $[19$, p. 245$]$ or by creating a nested FCM that decomposes the concepts in sub-concepts (e.g. "temperature" is decomposed in "high temperature" and "low temperature") shows a more differentiated system behavior [2, pp 15-17].

\section{5) Time-lags}

If respondents draw causal links with very different timeframes (e.g. A $\rightarrow$ B after a few days, B $\rightarrow$ C after 10 years) these time frames have to be synchronized through socalled "dummy concepts". These concepts are inserted in the more long-term causal link to break it up into several causal 
links with shorter time-frames [18]. In our case studies, however, we found that respondents chose fairly similar timeframes for all causal links so that synchronization was not required.

\section{B FCM-Modeling}

FCM-modeling is an iterative process that involves design and test of increasingly sophisticated models [8, pp 361-363]: in the beginning the refined causal maps are translated into a FCM. This conceptual FCM can be relatively crude and e.g. contain the same, simple activation function in all concepts and signed, but unweighted causal links. It can be tested by strongly varying the input states for the most active concepts and comparing the FCM result with the dynamic behavior expected by the experts. For large input variations, the conceptual FCM's dynamic behavior should match the behavior of the modeled system. If this is not the case, the modeler (and possibly also the experts) have to look for mistakes in the structure of the FCM, such as missing concepts and links in the FCM.

Once the concept FCM shows adequate system behavior in response to large variations, the FCM can be fine-tuned by applying fine-grained causal weights and choosing individual activation functions for each concept [8, pp 361-363]. Again, the testing of the system behavior is very important and a variety of testing approaches, adopted from System Dynamics and modified for use on FCM-models can be employed ([8, pp. 283-289]; adopted and modified from [23]), such as the Boundary Adequacy Test (a test for the adequacy of the problem framing), the Structure Assessment Test (a comparison of the model structure with the structure of the real-world problem), the Extreme Conditions Test (a test of the system behavior with extreme input values) and Sensitivity Analysis. Furthermore the general system behavior - the underlying meta-rules - can be experimentally identified and compared to the expected system behavior.

Because of cognitive limitations, it is well possible that experts are not able to correctly assess dynamic system behavior on the more detailed level of a parameterized FCM. Discrepancies between the model result and the result that the expert expects therefore have to be carefully analyzed and discussed with the experts who play an important role in the validation of the model, as the following section will show.

\section{TEST AND VALIDATION (STEP 6)}

Test and validation [8, pp 290-294] are not the final step, but ongoing activities in every FCM modeling project. They are challenging, because FCMs are based on experts' knowledge and worldviews and are oftentimes futureoriented. Consequently, external reference points rarely exist: A FCM that shows the influencing factors on an evolving technology, for example, can be used in technology forecasting. If the forecast turns out to be incorrect, there are two possible reasons for this: Firstly, the FCM could have been poorly designed and did not reflect the experts' knowledge, possibly because poor interviewing or cognitive mapping practice or because the modeler was not able to translate expert statements into an adequate FCM. Secondly, the experts could have been wrong and did not sufficiently understand the evolving technology or were not aware of an disruptive event like a scientific breakthrough that occurred after the FCM model was completed.

In most cases, it will be impossible to exactly pinpoint the causes for a failed FCM project because both problems can occur at the same time. Also fixing the FCM-model expost, so that it correctly reflects the development that has occurred in the real-world, is of little practical use: the modeling topic can be outdated (new and different technologies evolve) and even if the model is still of interest, there is no guarantee that it correctly forecasts the future (other aspects of the experts' worldviews could turn out to be wrong and new disruptive events can occur). As a consequence, several authors suggest to not search for "truth" in models, but for conceptual adequacy. "The question facing clients and modelers is never whether a model is true but whether it is useful. The choice is never whether to use a model. The only choice is which model to use. Selecting the most appropriate model is always a value judgment to be made by reference to the purpose" [23 p. 890].

Rather than focusing on external reference points for verification (e.g. historic data or data on related problems of the same problem family), modelers should therefore attempt to ensure model validity through a high quality modeling process in which every transformation step is validated in itself: This means that experts are thoroughly identified with the help of multiple references. Knowledge capture and the subsequent translation of experts' knowledge, first in causal maps and then in FCM-models need, to be well-prepared and systematically executed. Every step furthermore has to be validated through the experts, who have to agree that their mental models are correctly captured and through other available data (e.g. existing theories). Most importantly, the model has to be constantly tested against the behavior of the real-world system it represents. Any new "real-world" information must be assessed - if it does not fit the expectations, it should be used to challenge and improve the FCM-models.

\section{DISCUSSION AND CONCLUSION}

The purpose of this paper was to provided a framework and practical guideline for FCM modeling in order to close the "application gap" that has caused FCMs to remain a theoretical opportunity, rather than a useful decision-support and planning method, even though 20 years have passed since they were first described.

The presented approaches are founded on an extensive literature study and practice-tested and improved in several case studies and will therefore provide a useful help for practitioners and researchers who wish to use FCMs. They are, however, just this - a useful help, not a recipe for 
success: FCM-modelers will have to decide individually, which of the techniques to apply and how to adapt them to their specific problems. They will thus add to the body of knowledge about FCM-practice.

Better theoretical and practical knowledge about FCMs will help to reduce the application gap, but it will not suffice: in order to be adopted on a wider scale, user-friendly software for FCM modeling, testing and use is required that allows practitioners and research groups to tackle complex real-world problems that involve several experts and modelers and share results among them.

Adoption of FCMs can furthermore be improved through a better choice of applications: In the past, FCMs have been used for all kinds of problems and in some cases, the reason for choosing FCMs over other modeling techniques (e.g. System Dynamics or Bayesian networks) is all but clear. Future FCM research should focus on problems that FCMs are "good at": they are a powerful means to represent knowledge domains that are characterized by high complexity, by widespread knowledge sources that usually only have partial knowledge, by qualitative information that frequently changes, and by a lack of a commonly accepted "theory" or "truth". They can thus be useful for the analysis of business ecosystems, scenario planning, and the forecasting of market or technology trends and should be increasing applied in these areas.

Like many other models, e.g. System Dynamics models, they can help decision-makers to reflect upon their worldviews and to improve their understanding of the dynamic systems and decision alternative they encounter. Unlike these models, they can handle qualitative concepts with no dimensions and linguistic imprecision and so (relatively) simple to understand that they allow for a strong involvement of the decision-maker in modeling, simulation and interpretation of results. This leads to two research questions in the field of FCM-application:

- How can FCMs be practically applied in planning and decision-processes in Technology Management, what are the costs and efforts involved and what are the benefits? Up to now, we at best can answer these questions for isolated, one-time applications of FCMs (see e.g. Case Study 4 [8, pp. 376-435], which is an implementation of $[[7,20]$.

- Do Cognitive Mapping and the use of FCM-simulations improve decision-makers understanding of complex systems and their problem-framing and decision-making ability? Can FCM-modeling projects thus have an impact that reaches beyond the individual simulation model? Though there is some strong indication in cognitive psychology and the field of managerial cognition $[4 ; 8, \mathrm{pp}$. 223-247] that this could be the case, this has never been rigorously researched.

These questions can only be resolved by "leaving the desk" and applying FCMs in practice - FCMs today are theoretically mature enough to tackle them.

\section{REFERENCES}

[1] R. Axelrod Structure of Decision. The Cognitive Maps of Political Elites. Princeton: Princeton University Press, 1976.

[2] J. DiCkERSON and B. Kosko: "Virtual Worlds as Fuzzy Dynamical Systems" Technology for Multinedia B Sheu Ed IEEE Press 1996

[3] J. Hauschildt, "Methodische Anforderungen an die Ermittlung der Wissensbasis von Expertensystemen," Die Betriebswirtschaft, vol. 50, pp 525-537, 1990

[4] AS Huff Mapping Strategic Though. Chichester: John Wiley \& Sons, 1990

[5] J. P. Ignizio, An Introduction to Expert Systems: The Development and Implementation of Rule-Based Experts Systems New York: McGrawHill, 1991.

[6] AJ Jetter "Do maps guide the way to NPD success? Theoretical and practical aspects of knowledge mapping in product development" Technology Management: A Unifying Discipline for Melting the Boundaries. DF Kocaoglu TRAnderson Eds: Portland, OR 2005

[7] AJ Jetter "Educating the Guess: Strategies, Concepts and Tools for the Fuzzy Front End of Product Development" Technology Management for Reshaping the World Kocaoglu, D. F.; Anderson, T. R. Eds.: Portland, OR, pp.261-273, 2003.

[8] AJ Jetter Produktplanung im Fuzzy Front End: Handlungsunterstützung auf der Basis von Fuzzy Cognitive Maps Wiesbaden: Deutscher Universitätsverlag: 2005

[9] AJ Jetter, "Codification - Knowledge Maps," in Knowledge Integration: The Practice of Knowledge Management in Small and Medium Enterprises, A. J. Jetter, J. Kraaijenbrink, H.-H. Schröder, F. Wijnhoven Eds. Heidelberg, New York: Physica-Verlag, 2006

[10] AJ Jetter, "Elicitation - Extracting Knowledge from Experts," in Knowledge Integration: The Practice of Knowledge Management in Small and Medium Enterprises, A. J. Jetter, J. Kraaijenbrink, H.-H. Schröder, F. Wijnhoven Eds. Heidelberg, New York: Physica-Verlag, 2006

[11] D. Kardaras and B. Karakotas, "The use of fuzzy cognitive maps to simulate information systems strategic planning process," Information and Software Technology, vol. 41, pp. 197-210, 1999

[12] B. Kosko "Fuzzy cognitive maps" International Journal of ManMachine Studies Vol. 24 pp 65-75, 1986

[13] B. Kosko "Hidden patterns in combined and adaptive knowledge networks" International Journal of Approximative Reasoning, Vol. 2.pp 377-393, 1988

[14] B. Kosko "Adaptive Inference in Fuzzy Knowledge Networks," in Readings in Fuzzy Sets for Intelligent Systems D. Dubois, H. Prade Eds. San Mateo: Morgan Kaufman 1993

[15] S. Lee and I Han "Fuzzy cognitive map for the design of EDI controls" Information \& Management, Vol. 37, pp. 37-50, 2000

[16] Y Miao and Z-Q Liu "On Causal Inference in Fuzzy Cognitive Maps" IEEE Transactions on Fuzzy Systems Vol 8, pp 107-119, 2000

[17] S. Nadkarni and PP Shenoy "A causal mapping approach to constructing Bayesian networks" Decision Support Systems Vol 38, pp 259-281, 2004

[18] KS Park and SH Kim "Fuzzy Cognitive Maps considering time relationships" International Journal of Human-Computer Studies, Vol. 42, pp 157-168, 1995

[19] K. Perusich, M MoNeese, and J R Rentsch, "Qualitative modelling of complex systems for cognitive engineering" in Enabling Technology for Simulation Science III. Orlando, FL 6-8 April 1999 SPIE International Society for Optical Engineering Eds Vol. 3696 pp 240249 Orlando, FL 1999.

[20] H-H Schröder, AJ Jetter "Integrating market and technological knowledge in the fuzzy front-end: an FCM-based action support system" International Journal of Technology Management, Vol. 26, pp. 517-539, 2003.

[21] A. Sharif and Z. Irani "Fuzzy Cogntivie Mapping as a Technique for Technology Management”, PICMET 1997 Conference Proceedings, CD ROM, Portland OR 1997

[22] T. Smithin "Maps of the Mind: New Pathways to Decision-Making" Business Horizons, Vol.23, No.6, pp.24-28, December 1983 
PICMET 2006 Proceedings, 9-13 July, Istanbul, Turkey (c) 2006 PICMET

[23] J. D. Sterman, Business Dynamics. Systems Thinking and Modeling for a Complex World Boston: Irwin McGraw-Hill 2000.

[24] R. Taber "Fuzzy Cognitive Maps model social systems" Artificial Intelligence Expert, Vol. 9, pp. 18-23, 1994
[25] G. Xirogiannis and M. Glykas "Fuzzy Cognitive Maps in Business Analysis and Performance-Driven Change" IEEE Transactions on Engineering Management, Vol 51, No 3, August 2004

[26] H-J Zimmermann Fuzzy Set Theory - and its Applications: $4^{\text {th }}$ edition Dordrecht: Kluwer, 2001 